\title{
Relationships between cystatin C- and creatinine-based eGFR in Japanese rural community- dwelling older adults with sarcopenia
}

\author{
Hiroshi Kusunoki ${ }^{1}$. Shotaro Tsuji ${ }^{2} \cdot$ Tomoyuki Kusukawa $^{2} \cdot$ Yosuke Wada $^{3,4} \cdot$ Kayoko Tamaki $^{1} \cdot$ Koutatsu Nagai $^{5}$. \\ Masako Itoh ${ }^{5} \cdot K$ yoko Sano ${ }^{5}$ Manabu Amano ${ }^{6} \cdot$ Hatsuo Maeda $^{6} \cdot$ Hideyuki Sugita $^{7} \cdot$ Yoko Hasegawa $^{7,8}$. \\ Hiromitsu Kishimoto ${ }^{7} \cdot$ Soji Shimomura ${ }^{3} \cdot$ Ken Shinmura ${ }^{1,3}$
}

Received: 8 April 2020 / Accepted: 5 October 2020 / Published online: 22 October 2020

(c) The Author(s) 2020

\begin{abstract}
Background Sarcopenia is prevalent in patients with chronic kidney disease (CKD). The indices of physical function, such as grip power and gait speed, decreased according to the decline in the estimated glomerular filtration rate (eGFR).

Methods We examined the relationships between cystatin C-based GFR (eGFRcys), creatinine-based GFR (eGFRcre), their ratio (eGFRcys/eGFRcre) and sarcopenia in community-dwelling older adults in Japan. This cross-sectional study included 302 men aged $73.9 \pm 6.2$ years and 647 women aged $72.9 \pm 5.8$ years from a rural area in Hyogo Prefecture, Japan. eGFRcys and eGFRcre were simultaneously measured, and sarcopenia based on the Asia Working Group for Sarcopenia (AWGS) 2019 criteria was evaluated.

Results eGFRcys and the eGFRcys/eGFRcre ratio were significantly correlated with grip power and gait speed $(p<0.001)$. The eGFRcys/eGFRcre ratio was also correlated with skeletal muscle mass index (SMI) $(p<0.01)$. Univariate logistic regression analysis showed eGFRcys and eGFRcys/eGFRcre ratio but not eGFRcre were associated with sarcopenia $(p<0.01)$. The presence of low eGFRcys (CKDcys) and low eGFRcys/eGFRcre ratio $(<1.0)$ but not that of low eGFRcre (CKDcre) were associated with sarcopenia $(p<0.01)$. In the multivariate logistic regression analysis, when the eGFRcys/eGFRcre ratio was added as a covariate to the basic model, it was significantly associated with sarcopenia in women $(p<0.05)$. Moreover, low eGFRcys/eGFRcre ratio $(<1.0)$ was associated with a higher risk of sarcopenia in men $(p<0.01)$.

Conclusion In conclusion, CKDcys but not CKDcre is associated with sarcopenia. A lower eGFRcys/eGFRcre ratio may be a practical screening marker of sarcopenia in community-dwelling older adults.
\end{abstract}

Keywords Sarcopenia $\cdot$ eGFR $\cdot$ AWGS $\cdot$ Cystatin C $\cdot$ Skeletal muscle mass index (SMI)

$\begin{array}{llll}\text { Abbreviations } & \text { CysC } & \text { Cystatin C } \\ \text { CKD } & \text { Chronic kidney disease } & \text { BMI } & \text { Body mass index } \\ \text { eGFR } & \text { Estimated glomerular filtration rate } & \text { OR } & \text { Odds ratio } \\ \mathrm{Cr} & \text { Creatinine } & \text { CI } & \text { Confidence interval }\end{array}$

Ken Shinmura

ke-shimmura@hyo-med.ac.jp

1 Division of General Medicine, Department of Internal Medicine, Hyogo College of Medicine, 1-1 Mukogawa-cho, Nishinomiya, Hyogo 663-8501, Japan

2 Department of Orthopaedic Surgery, Hyogo College of Medicine, Nishinomiya, Hyogo, Japan

3 Department of General Medicine and Community Health Science, Sasayama Medical Center Hyogo College of Medicine, Sasayama, Hyogo, Japan

4 Department of Rehabilitation Medicine, Sasayama Medical Center Hyogo College of Medicine, Sasayama, Hyogo, Japan
5 School of Rehabilitation, Hyogo University of Health Sciences, Kobe, Hyogo, Japan

6 School of Pharmacy, Hyogo University of Health Sciences, Kobe, Hyogo, Japan

7 Department of Dentistry and Oral Surgery, Hyogo College of Medicine, Nishinomiya, Hyogo, Japan

8 Division of Comprehensive Prosthodontics, Niigata University Graduate School of Medical and Dental Sciences, Niigata, Niigata, Japan 


$\begin{array}{ll}\text { SD } & \text { Standard deviations } \\ \text { FESTA } & \text { Frail Elderly in Sasayama-Tamba Area } \\ \text { KDIGO } & \text { Kidney Disease: Improving Global Outcomes } \\ \text { AWGS } & \text { Asian Working Group for Sarcopenia } \\ \text { BIA } & \text { Bioelectrical impedance analysis } \\ \text { SMM } & \text { Skeletal muscle mass } \\ \text { SMI } & \text { Skeletal muscle mass index } \\ \text { BFM } & \text { Body fat mass } \\ \text { CVDs } & \text { Cardiovascular diseases } \\ \text { Hb } & \text { Hemoglobin } \\ \text { Alb } & \text { Albumin } \\ \text { ROC } & \text { Receiver operating characteristic curve } \\ \text { AUC } & \text { Area under the curve }\end{array}$

\section{Introduction}

Sarcopenia is a disease characterized by loss of skeletal muscle mass (SMM), and it is an important public health problem. Sarcopenia is common in patients with chronic kidney disease (CKD). The muscle strength, for example grip power and physical function such as gait speed, decreased with the decline in the estimated glomerular filtration rate (eGFR) [1]. Serum creatinine $(\mathrm{Cr})$ is a common biomarker that reflects not only renal function, but also systemic muscle mass. Cystatin $\mathrm{C}(\mathrm{Cys} \mathrm{C})$ may be a more reliable biomarker that estimates the glomerular filtration rate (eGFR), and it is not influenced by sex, age, or muscle mass. CKD is defined as reduced eGFR. We previously reported that the $\mathrm{Cr} / \mathrm{CysC}$ ratio was positively correlated to muscle volume and physical function [2]. Another study has reported that the $\mathrm{Cr} / \mathrm{CysC}$ ratio was associated with high risk of sarcopenia [3]. A declined eGFR based on CysC (eGFRcys) was associated with a higher prevalence and incidence of frailty, whereas eGFR based on creatinine (eGFRcre) was not [4]. eGFRcys is related to a higher risk of sarcopenia than eGFRcre, because eGFRcys is not influenced by low muscle mass or quality [5].

Kurajoh et al. have reported that a low eGFRcys (CKDcys), but not a low eGFRcre (CKDcre), was independently related to osteoporotic fracture in postmenopausal women. Furthermore, the eGFRcys/eGFRcre ratio was independently related to osteoporotic fracture in this study and was correlated to physical function. The eGFRcys/eGFRcre ratio may be a clinically useful parameter for loss of muscle mass [6]. Several studies have shown the correlation between sarcopenia and osteoporosis [7, 8]. and have suggested a significant correlation between bone and muscle as well as sarcopenia and osteoporosis. Moreover, a prospective study has found that patients with osteoporosis are at an increased risk of developing sarcopenia [9].

We hypothesized that eGFRcys is superior to eGFRcre in evaluating muscle mass and physical function, and is more associated with sarcopenia. The eGFRcys/eGFRcre ratio may provide a clinically relevant measurement of muscle mass. eGFRcre is determined by not only renal function but also muscle mass, thus indicating that a low eGFRcys/ eGFRcre ratio is a clinically useful parameter for sarcopenia, because eGFRcre is likely to be overestimated and larger than eGFRcys in sarcopenia [5]. We hypothesized that a eGFRcys/eGFRcre ratio $<1.0$ can be related to sarcopenia. Hence, we examined the relationship between eGFRcys and eGFRcre, as well as the relationship between the eGFRcys/ eGFRcre ratio and sarcopenia in community-dwelling older adults in Japan.

\section{Materials and methods}

This cross-sectional study was called the Frail Elderly in Sasayama-Tamba Area (FESTA) study. The study population was composed of individuals aged $\geq 65$ years. Healthy community-dwelling elderly individuals from the SasayamaTamba area, a rural area in Hyogo Prefecture, Japan, were recruited between 2015 and 2019. Physical function assessment, measurement of body composition, and blood sample analysis were performed as described previously [2].

\section{Categorization of CKD}

CKD was defined and classified according to the Kidney Disease: Improving Global Outcomes (KDIGO) criteria [10]. eGFRcre and eGFRcys were calculated using equations by the Japanese Society of Nephrology $[11,12]$. Low eGFRcre (CKDcre) is defined as an eGFRcre $<60 \mathrm{~mL} /$ $\mathrm{min} / 1.73 \mathrm{~m}^{2}$. Low eGFRcys (CKDcys) is defined as an eGFRcys $<60 \mathrm{~mL} / \mathrm{min} / 1.73 \mathrm{~m}^{2}$.

\section{Diagnosis of sarcopenia}

Sarcopenia was defined according to the criteria for the Asia Working Group for Sarcopenia (AWGS) 2019 [13]. Body composition was evaluated by bioelectrical impedance analysis (BIA) using an InBody $770^{\circledR}$ (InBody Japan Inc., Tokyo, Japan). The skeletal muscle mass index (SMI) was calculated as SMM/height ${ }^{2}\left(\mathrm{~kg} / \mathrm{m}^{2}\right)$. The handgrip power, and the normal and maximal gait speed, 5-time chair stand test (5CS), Timed Up and Go test (TUG), and Short Physical Performance Battery (SPPB) scores were evaluated as described previously [2,13]. Sarcopenia was considered if the participants had a low SMI $\left(<7.0 \mathrm{~kg} / \mathrm{m}^{2}\right.$ in men; $<5.7 \mathrm{~kg} / \mathrm{m}^{2}$ in women) and weak handgrip strength $(<28 \mathrm{~kg}$ in men; $<18 \mathrm{~kg}$ in women) or low physical 
performance (normal gait speed $<1.0 \mathrm{~m} / \mathrm{s}, 5 \mathrm{CS} \geq 12 \mathrm{~s}$ or $\mathrm{SPPB} \leq 9)$.

\section{Statistical analysis}

The results are expressed as mean \pm standard deviations (SD) or percentages. For intergroup comparisons, the student's $t$ test was used for data analysis. Pearson's product moment correlation coefficient was used to assess the associations between eGFRcre, eGFRcys and the eGFRcys/eGFRcre ratio and SMM, SMI, body Fat mass (BFM), percentage of BFM, grip power, knee extension muscle strength, normal gait speed, maximal gait speed, and TUG and 5CS scores. Categorical variables were expressed as absolute $(n)$ and relative frequency (\%) and analyzed by Fisher's exact test. Univariate and multivariate logistic regression analysis was performed to calculate the odds ratio and $95 \%$ confidence interval. A receiver operating characteristic curve (ROC) analysis was performed to confirm the diagnostic efficacy of the eGFRcys/eGFRcre, and the area under the curve (AUC) was calculated. For data analysis, the JMP 13.1 software was used. $p$ values $<0.05$ were considered significant.

\section{Results}

The baseline characteristics, indices of body composition, and physical performance of the participants are presented in Table 1. The study included 302 men aged 65-94 years, and 647 women aged 65-91 years. The BFM weight and BFM percentages (BFM \%) were higher in women than in men. Maximal gait speed, grip power, knee extension muscle strength, SMM, and SMI were higher in men than in women $(p<0.001)$ (Table 1$)$. The average eGFRcre was 69.0 (men: 68.1, and women: 69.3). The average eGFRcys

Table 1 Clinical characteristics, body composition, and physical performance in the subjects

\begin{tabular}{|c|c|c|c|c|}
\hline & Total $(n=949)$ & Men $(n=302)$ & Women $(n=647)$ & $p$ value \\
\hline Age (year-old) & $73.2 \pm 5.9$ & $73.9 \pm 6.2$ & $72.9 \pm 5.8$ & 0.014 \\
\hline Height $(\mathrm{cm})$ & $155.3 \pm 8.1$ & $163.9 \pm 5.9$ & $151.3 \pm 5.6$ & $<0.001$ \\
\hline Body weight (kg) & $54.9 \pm 9.3$ & $62.6 \pm 9.0$ & $51.4 \pm 7.0$ & $<0.001$ \\
\hline Body mass index & $22.7 \pm 2.9$ & $23.3 \pm 2.9$ & $22.4 \pm 2.8$ & $<0.001$ \\
\hline Skeletal muscle mass (SMM) $(\mathrm{kg})$ & $15.7 \pm 3.7$ & $19.9 \pm 2.7$ & $13.7 \pm 1.9$ & $<0.001$ \\
\hline Skeletal muscle mass index (SMI) & $6.41 \pm 0.93$ & $7.39 \pm 0.71$ & $5.96 \pm 0.61$ & $<0.001$ \\
\hline Body fat mass (kg) & $15.5 \pm 5.4$ & $15.0 \pm 5.7$ & $15.8 \pm 5.2$ & 0.026 \\
\hline Percentage of BFM (\%) & $28.0 \pm 7.6$ & $23.4 \pm 6.7$ & $30.2 \pm 7.0$ & $<0.001$ \\
\hline Grip power $(\mathrm{kg})$ & $27.0 \pm 7.3$ & $34.9 \pm 6.2$ & $23.4 \pm 4.3$ & $<0.001$ \\
\hline Knee extension muscle strength (Nm) & $348.7 \pm 118.5$ & $450.6 \pm 121.1$ & $301.1 \pm 81.5$ & $<0.001$ \\
\hline Normal gait speed (m/s) & $1.47 \pm 0.24$ & $1.45 \pm 0.25$ & $1.48 \pm 0.24$ & 0.073 \\
\hline Maximal gait speed (m/s) & $1.90 \pm 0.31$ & $1.96 \pm 0.33$ & $1.87 \pm 0.30$ & $<0.001$ \\
\hline Timed Up and Go test (TUG) & $6.27 \pm 1.52$ & $6.25 \pm 1.85$ & $6.28 \pm 1.34$ & 0.825 \\
\hline 5-time chair stand test (5CS) & $7.73 \pm 4.26$ & $8.21 \pm 4.79$ & $7.50 \pm 3.99$ & 0.018 \\
\hline Short physical performance battery (SPPB) $\leqq 9, n(\%)$ & $17(1.8)$ & $10(3.3)$ & $7(1.1)$ & 0.795 \\
\hline Cre $(\mathrm{mg} / \mathrm{dL})$ & $0.72 \pm 0.18$ & $0.87 \pm 0.12$ & $0.65 \pm 0.13$ & $<0.001$ \\
\hline CysC (mg/dL) & $0.94 \pm 0.20$ & $1.01 \pm 0.22$ & $0.90 \pm 0.18$ & $<0.001$ \\
\hline eGFRcre $\left(\mathrm{mL} / \mathrm{min} / 1.73 \mathrm{~m}^{2}\right)$ & $69.0 \pm 13.7$ & $68.1 \pm 13.5$ & $69.3 \pm 13.8$ & 0.205 \\
\hline eGFRcys (mL/min/1.73 m²) & $74.1 \pm 15.6$ & $71.7 \pm 15.9$ & $75.3 \pm 15.3$ & 0.001 \\
\hline eGFRcys/eGFRcre & $1.08 \pm 0.17$ & $1.06 \pm 0.17$ & $1.10 \pm 0.17$ & 0.006 \\
\hline Sarcopenia, $n(\%)$ & $75(7.9)$ & $25(8.3)$ & $50(7.7)$ & 0.797 \\
\hline Hypertension, $n(\%)$ & $418(44.0)$ & $149(49.3)$ & 269 (41.6) & 0.030 \\
\hline Diabetes, $n(\%)$ & $112(11.8)$ & $56(18.5)$ & $56(8.7)$ & $<0.001$ \\
\hline Dyslipidemia, $n(\%)$ & $213(22.4)$ & $51(16.9)$ & $162(25.0)$ & 0.006 \\
\hline Liver disease, $n(\%)$ & $38(4.0)$ & $17(5.6)$ & $21(3.2)$ & 0.108 \\
\hline Heart disease, $n(\%)$ & $63(6.6)$ & $31(10.3)$ & $32(4.9)$ & 0.003 \\
\hline CKDcre (eGFRcre $\left.<60 \mathrm{~mL} / \mathrm{min} / 1.73 \mathrm{~m}^{2}\right), n(\%)$ & $225(23.7)$ & $79(26.2)$ & $146(22.6)$ & 0.251 \\
\hline CKDcys (eGFRcys $\left.<60 \mathrm{~mL} / \mathrm{min} / 1.73 \mathrm{~m}^{2}\right), n(\%)$ & $168(17.7)$ & $70(23.2)$ & $98(15.1)$ & 0.003 \\
\hline eGFRcys/eGFRcre $<1.0, n(\%)$ & $319(33.6)$ & $121(40.1)$ & $198(30.6)$ & 0.005 \\
\hline
\end{tabular}

Data are expressed as mean $\pm \mathrm{SD}$ 
was $74.1 \mathrm{~mL} / \mathrm{min} / 1.73 \mathrm{~m}^{2}$ (men: $71.7 \mathrm{~mL} / \mathrm{min} / 1.73 \mathrm{~m}^{2}$, and women: $75.3 \mathrm{~mL} / \mathrm{min} / 1.73 \mathrm{~m}^{2}$ ).

Among the 949 participants, 75 ( 25 men and 50 women) participants had sarcopenia based on the AWGS 2019 criteria (Table 1). Other complications (hypertension, diabetes, dyslipidemia, and heart disease) were more prevalent in men than in women $(p<0.05)$. In total, 225 (79 men and 146 women) participants had low eGFRcre (CKDcre), and 168 (70 men and 98 women) had low eGFRcys (CKDcys). In total, 319 (121 men and 198 women) participants had a low eGFRcys/eGFRcre ratio $(<1.0)$.

The characteristics of all participants are shown in Table 2. Among both men and women, participants with sarcopenia were older than normal participants $(p<0.001)$. Height, body weight, and body mass index (BMI) were lower in participants with sarcopenia than in normal participants, in both men and women $(p<0.001)$. Similarly, normal and maximal gait speed decreased in the participants with sarcopenia $(p<0.001)$. In participants with sarcopenia, muscle strength (grip power and knee extension muscle strength) and muscle volume (SMM and SMI) were lower than in normal participants, in both men and women $(p<0.001)$. TUG and 5CS scores were higher in participants with sarcopenia than in normal participants, irrespective of sex $(p<0.01)$. Body fat mass (BFM) was also lower in participants with sarcopenia than in normal participants, in both men and women $(p<0.05)$, however, there was no significant difference in percentage of BFM between men and women. While there is no difference in Cre value between normal and sarcopenia, CysC value was significantly higher (more than $10 \%)$ in sarcopenia subjects in both sexes $(p<0.01)$. eGFRcys but not eGFRcre decreased in both men and women with sarcopenia $(p<0.01)$. The eGFRcys/eGFRcre ratio also decreased in all participants with sarcopenia $(p<0.01)$.

The correlations between eGFRcre, eGFRcys, and the eGFRcys/eGFRcre ratio and the parameters of body composition based on bioelectrical impedance analysis (BIA) (such as SMI, SMM, BFM, and percentage of BFM) and muscle strength and physical function parameters (grip power, knee extension muscle strength, normal gait speed, and maximal gait speed, TUG, 5CS) are shown in Table 3. eGFRcre was not significantly correlated to muscle volume and strength parameters. On the

Table 2 Characteristics of subjects with and without sarcopenia

\begin{tabular}{|c|c|c|c|c|c|c|}
\hline & \multicolumn{3}{|l|}{ Men } & \multicolumn{3}{|l|}{ Women } \\
\hline & Normal $(n=277)$ & Sarcopenia $(n=25)$ & $p$ value & Normal $(n=597)$ & Sarcopenia $(n=50)$ & $p$ value \\
\hline Age (year-old) & $73.4 \pm 5.9$ & $77.7 \pm 7.9$ & 0.001 & $72.5 \pm 5.6$ & $77.5 \pm 5.9$ & $<0.001$ \\
\hline Height $(\mathrm{cm})$ & $164.3 \pm 5.8$ & $158.9 \pm 5.0$ & $<0.001$ & $151.8 \pm 5.3$ & $145.6 \pm 5.4$ & $<0.001$ \\
\hline Body weight (kg) & $63.6 \pm 8.5$ & $51.9 \pm 16.9$ & $<0.001$ & $51.9 \pm 6.9$ & $44.6 \pm 5.2$ & $<0.001$ \\
\hline Body mass index & $23.5 \pm 2.8$ & $20.5 \pm 2.5$ & $<0.001$ & $22.5 \pm 2.8$ & $21.0 \pm 2.5$ & $<0.001$ \\
\hline Skeletal muscle mass (SMM) $(\mathrm{kg})$ & $20.3 \pm 2.5$ & $15.9 \pm 1.6$ & $<0.001$ & $13.9 \pm 1.8$ & $10.9 \pm 1.3$ & $<0.001$ \\
\hline Skeletal muscle mass index (SMI) & $7.49 \pm 0.64$ & $6.29 \pm 0.46$ & $<0.001$ & $6.02 \pm 0.58$ & $5.15 \pm 0.36$ & $<0.001$ \\
\hline Body fat mass (kg) & $15.2 \pm 5.7$ & $12.6 \pm 5.2$ & 0.028 & $16.0 \pm 5.2$ & $13.5 \pm 4.4$ & 0.011 \\
\hline Percentage of BFM (\%) & $23.4 \pm 5.7$ & $23.6 \pm 7.5$ & 0.867 & $30.2 \pm 7.0$ & $29.7 \pm 7.0$ & 0.615 \\
\hline Grip power $(\mathrm{kg})$ & $35.7 \pm 5.7$ & $26.3 \pm 4.1$ & $<0.001$ & $24.0 \pm 3.9$ & $16.3 \pm 3.1$ & $<0.001$ \\
\hline Knee extension muscle strength $(\mathrm{Nm})$ & $462.1 \pm 117.6$ & $323.2 \pm 80.5$ & $<0.001$ & $307.5 \pm 77.4$ & $224.6 \pm 70.1$ & $<0.001$ \\
\hline Normal gait speed $(\mathrm{m} / \mathrm{s})$ & $1.46 \pm 0.24$ & $1.28 \pm 0.25$ & $<0.001$ & $1.49 \pm 0.23$ & $1.31 \pm 0.34$ & $<0.001$ \\
\hline Maximal gait speed (m/s) & $1.98 \pm 0.33$ & $1.73 \pm 0.27$ & $<0.001$ & $1.89 \pm 0.29$ & $1.62 \pm 0.31$ & $<0.001$ \\
\hline Timed Up and Go test (TUG) & $6.16 \pm 1.79$ & $7.31 \pm 2.19$ & 0.003 & $6.02 \pm 0.58$ & $7.41 \pm 2.08$ & $<0.001$ \\
\hline 5-time chair stand test (5CS) & $8.00 \pm 4.12$ & $10.85 \pm 9.72$ & 0.007 & $7.23 \pm 2.45$ & $10.73 \pm 11.20$ & $<0.001$ \\
\hline $\begin{array}{l}\text { Short physical performance battery } \\
(\text { SPPB }) \leqq 9, n(\%)\end{array}$ & $4(1.4)$ & $2(8.0)$ & $<0.001$ & $6(1.0)$ & $5(10.0)$ & $<0.001$ \\
\hline Cre (mg/dL) & $0.87 \pm 0.17$ & $0.90 \pm 0.19$ & 0.374 & $0.65 \pm 0.13$ & $0.66 \pm 0.14$ & 0.670 \\
\hline CysC (mg/dL) & $1.00 \pm 0.21$ & $1.13 \pm 0.26$ & 0.003 & $0.89 \pm 0.17$ & $0.97 \pm 0.24$ & 0.004 \\
\hline eGFRcre $\left(\mathrm{mL} / \mathrm{min} / 1.73 \mathrm{~m}^{2}\right)$ & $68.4 \pm 13.6$ & $65.0 \pm 13.2$ & 0.230 & $69.5 \pm 13.8$ & $67.6 \pm 14.1$ & 0.339 \\
\hline eGFRcys $\left(\mathrm{mL} / \mathrm{min} / 1.73 \mathrm{~m}^{2}\right)$ & $72.5 \pm 15.5$ & $62.7 \pm 16.9$ & 0.003 & $75.8 \pm 15.1$ & $69.1 \pm 17.1$ & $<0.001$ \\
\hline eGFRcys/eGFRcre & $1.07 \pm 0.17$ & $0.96 \pm 0.14$ & 0.002 & $1.10 \pm 0.17$ & $1.02 \pm 0.14$ & $<0.001$ \\
\hline $\operatorname{CKD} c r e(+), n(\%)$ & $70(25.2)$ & $9(36.0)$ & 0.242 & $131(21.9)$ & $15(30.0)$ & 0.217 \\
\hline CKDcys $(+), n(\%)$ & $57(20.6)$ & $13(52.0)$ & 0.001 & $83(13.9)$ & $15(30.0)$ & 0.006 \\
\hline eGFRcys/eGFRcre $<1.0, n(\%)$ & $104(37.5)$ & 17 (68.0) & 0.005 & $5177(29.6)$ & $21(42.0)$ & 0.079 \\
\hline
\end{tabular}

Data are expressed as mean $\pm \mathrm{SD}$ 
Table 3 Correlations between eGFRcre, eGFRcys and eGFRcys/eGFRcre and the parameters of body composition based on BIA, muscle strength and physical function parameters

\begin{tabular}{|c|c|c|c|c|c|c|c|c|c|c|c|c|}
\hline & \multicolumn{6}{|c|}{ Men $(n=302)$} & \multicolumn{6}{|c|}{ Women $(n=647)$} \\
\hline & \multicolumn{2}{|c|}{ eGFRcre } & \multicolumn{2}{|c|}{ eGFRcys } & \multicolumn{2}{|c|}{$\begin{array}{l}\text { eGFRcys/eGFR- } \\
\text { cre }\end{array}$} & \multicolumn{2}{|c|}{ eGFRcre } & \multicolumn{2}{|c|}{ eGFRcys } & \multicolumn{2}{|c|}{$\begin{array}{l}\text { eGFRcys/eGFR- } \\
\text { cre }\end{array}$} \\
\hline & $\mathrm{r}$ & $p$ value & $r$ & $p$ value & $r$ & $p$ value & $r$ & $p$ value & $r$ & $p$ value & $r$ & $p$ value \\
\hline $\begin{array}{l}\text { Skeletal muscle mass index } \\
\text { (SMI) }\end{array}$ & -0.08 & 0.164 & 0.13 & 0.026 & 0.29 & $<0.001$ & -0.02 & 0.631 & 0.05 & 0.172 & 0.12 & 0.003 \\
\hline Skeletal muscle mass (SMM) & -0.04 & 0.446 & 0.13 & 0.023 & 0.26 & $<0.001$ & 0.02 & 0.638 & 0.13 & $<0.001$ & 0.17 & $<0.001$ \\
\hline Body fat mass & -0.13 & 0.024 & -0.25 & $<0.001$ & -0.18 & 0.001 & -0.02 & 0.671 & -0.17 & $<0.001$ & -0.19 & $<0.001$ \\
\hline Percentage of BFM & -0.13 & 0.023 & -0.29 & $<0.001$ & -0.25 & $<0.001$ & -0.02 & 0.632 & -0.18 & $<0.001$ & -0.21 & $<0.001$ \\
\hline Grip power & -0.06 & 0.293 & 0.24 & $<0.001$ & 0.43 & $<0.001$ & 0.03 & 0.420 & 0.21 & $<0.001$ & 0.25 & $<0.001$ \\
\hline Knee extension muscle strength & 0.06 & 0.305 & 0.28 & $<0.001$ & 0.34 & $<0.001$ & 0.05 & 0.206 & 0.19 & $<0.001$ & 0.20 & $<0.001$ \\
\hline Normal gait speed & 0.11 & 0.067 & 0.24 & $<0.001$ & 0.20 & $<0.001$ & 0.10 & 0.011 & 0.23 & $<0.001$ & 0.19 & $<0.001$ \\
\hline Maximal gait speed & 0.11 & 0.053 & 0.29 & $<0.001$ & 0.27 & $<0.001$ & 0.08 & 0.040 & 0.27 & $<0.001$ & 0.27 & $<0.001$ \\
\hline Timed up and go test (TUG) & -0.16 & 0.005 & -0.39 & $<0.001$ & -0.36 & $<0.001$ & -0.12 & 0.003 & -0.27 & $<0.001$ & -0.23 & $<0.001$ \\
\hline 5-time chair stand test (5CS) & -0.03 & 0.583 & -0.10 & 0.078 & -0.11 & 0.050 & 0.03 & 0.445 & -0.06 & 0.121 & -0.13 & $<0.001$ \\
\hline
\end{tabular}

contrary, eGFRcys was positively correlated to SMM and muscle strength parameters in all participants $(p<0.05)$ and was negatively correlated to BFM and percentage of BFM $(p<0.001)$. The eGFRcys/eGFRcre ratio was significantly correlated to SMI, SMM, and muscle strength parameters and exhibited a negative correlation with $\mathrm{BFM}$, percentage of BFM and, TUG and 5CS scores $(p<0.05)$.

Figure 1 shows the ROC curves of eGFRcys/eGFRcre for identifying sarcopenia in men (A) and women (B). The AUC of eGFRcys/eGFRcre was 0.693 in men and 0.630 in women. The cut-off value was 1.00 in men and 1.09 in women (Fig. 1).

Univariate logistic regression analysis showed eGFRcys and eGFRcys/eGFRcre but not eGFRcre were associated with sarcopenia $(p<0.01)$. Moreover presence of low eGFRcys (CKDcys) and low eGFRcys/eGFRcre ratio $(<1.0)$ but not that of low eGFRcre (CKDcre) were associated with sarcopenia $(p<0.01)$ (Table 4$)$.

Multivariate logistic regression analysis was performed to examine whether eGFRcys, eGFRcre, and the eGFRcys/eGFRcre ratio were independently associated with sarcopenia. Although eGFRcys was not significantly associated with sarcopenia, when the eGFRcys/eGFRcre ratio was added as a covariate to the basic model, a significant association was observed between the eGFRcys/ eGFRcre ratio and sarcopenia in women $(p<0.05)$ (Table 5).

Multivariate logistic regression analysis was performed adjusted for complications (hypertension, diabetes, dyslipidemia, liver disease, and heart disease) to examine whether CKDcys and CKDcre were independently associated with sarcopenia. CKDcys was independently associated with sarcopenia both in men and women and an eGFRcys/eGFRcre $<1.0$ was independently associated with sarcopenia in men $(p<0.01)$ (Table 6).

\section{Discussion}

The present study showed that, low eGFRcys (CKDcys) was more frequent in participants with sarcopenia than in normal participants (Table 2). However there was no difference in the frequency of low eGFRcre (CKDcre) between sarcopenia and normal participants. In the multivariate logistic regression analysis adjusted for complications (hypertension, diabetes, dyslipidemia, liver disease, and heart disease), CKDcys was clearly related to sarcopenia based on AWGS 2019 while CKDcre was not (Table 6). CKD is considered a risk factor for several aging-related diseases, including cardiovascular diseases (CVDs), metabolic syndrome, frailty, and sarcopenia. Previous studies have reported that CKDcys was more associated with life prognosis and physical function than CKDcre. In octogenarians, CKDcys was associated with increased odds of CVDs [14]. The Cardiovascular Health Study has shown that elderly individuals with CKDcys had a high risk of heart failure and mortality [15]. In the Framingham Offspring Study, participants with CKDcys had greater gait speed declines than those with CKDcre. The participants with CKDcys also had greater odds of mobility disability than those with CKDcre [16]. These previous reports support our result that, CKDcys is more associated with sarcopenia than CKDcre. 

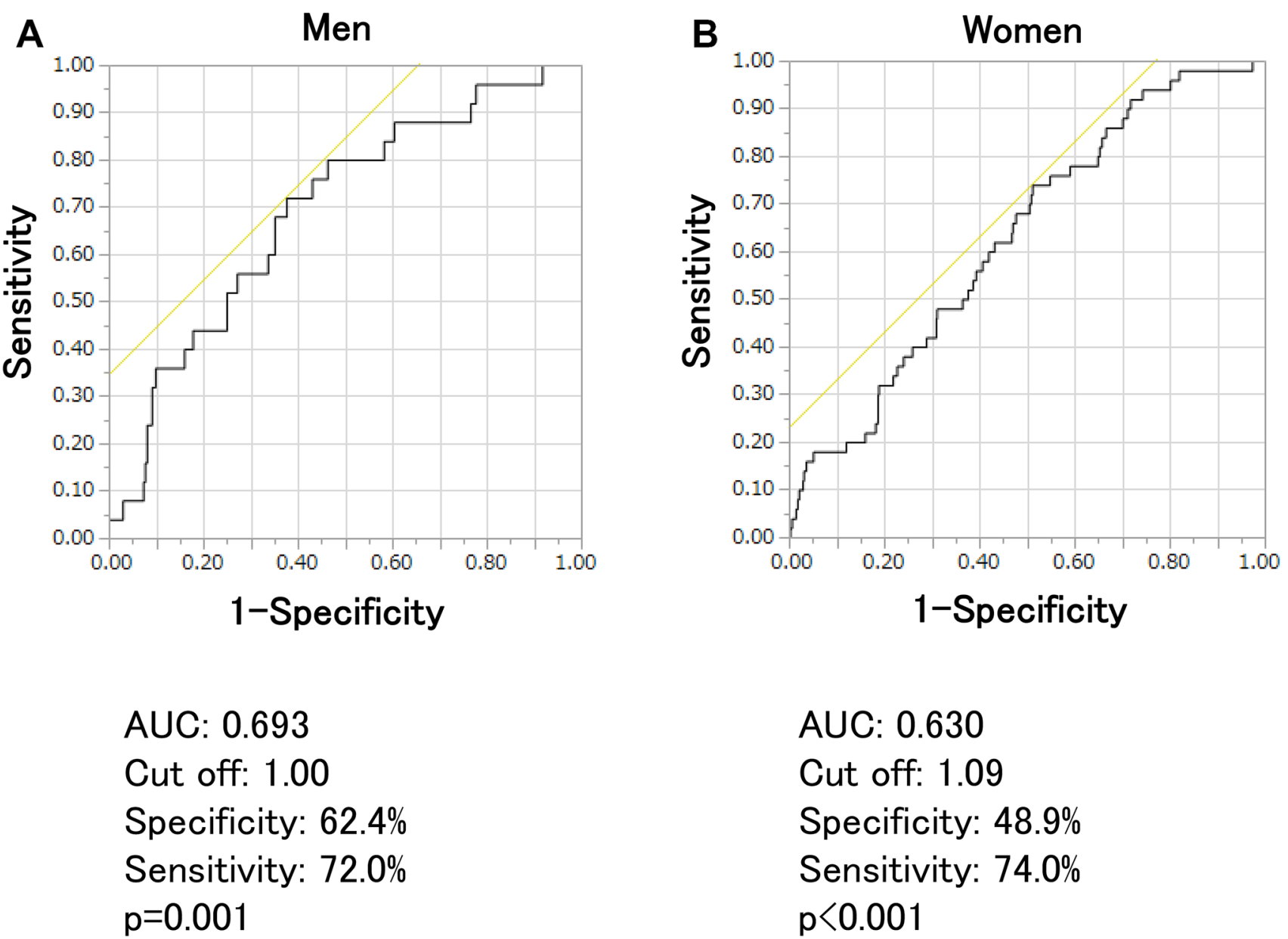

Fig. 1 Receiver operating characteristic curves for eGFRcys/eGFRcre ratio and sarcopenia based on the AWGS 2019 criteria

Table 4 Univariate logistic regression analysis of factors associated with sarcopenia in men and women

\begin{tabular}{|c|c|c|c|c|}
\hline \multirow[t]{2}{*}{ Variables } & \multicolumn{2}{|l|}{ Men } & \multicolumn{2}{|l|}{ Women } \\
\hline & OR $(95 \% \mathrm{CI})$ & $p$ value & OR $(95 \% \mathrm{CI})$ & $p$ value \\
\hline Age (per 1SD) & $1.85(1.25-2.75)$ & 0.002 & $2.22(1.67-2.95)$ & $<0.001$ \\
\hline BMI (per 1SD) & $0.25(0.14-0.44)$ & $<0.001$ & $0.55(0.40-0.77)$ & $<0.001$ \\
\hline $\mathrm{Hb}$ (per 1SD) & $0.72(0.53-0.96)$ & 0.026 & $0.91(0.72-1.14)$ & 0.421 \\
\hline Alb (per 1SD) & $0.40(0.26-0.61)$ & $<0.001$ & $0.83(0.61-1.12)$ & 0.226 \\
\hline eGFRcre (per 1SD) & $0.77(0.51-1.18)$ & 0.225 & $0.87(0.65-1.16)$ & 0.337 \\
\hline eGFRcys (per 1SD) & $0.53(0.35-0.82)$ & 0.003 & $0.65(0.48-0.86)$ & 0.003 \\
\hline eGFRcys/eGFRcre (per 1SD) & $0.48(0.30-0.77)$ & 0.001 & $0.58(0.41-0.80)$ & $<0.001$ \\
\hline Hypertension $($ absence $=0$, presence $=1)$ & $0.66(0.29-1.52)$ & 0.328 & $1.02(0.57-1.83)$ & 0.950 \\
\hline Diabetes $($ absence $=0$, presence $=1)$ & $0.58(0.17-2.00)$ & 0.356 & $0.91(0.32-2.63)$ & 0.862 \\
\hline Dyslipidemia $($ absence $=0$, presence $=1)$ & $0.40(0.09-1.77)$ & 0.179 & $0.31(0.12-0.80)$ & 0.005 \\
\hline Liver disease $($ absence $=0$, presence $=1)$ & $1.52(0.327-7.05)$ & 0.610 & $1.27(0.29-5.60)$ & 0.762 \\
\hline Heart disease $($ absence $=0$, presence $=1)$ & $2.41(0.84-6.97)$ & 0.128 & $2.35(0.86-6.38)$ & 0.125 \\
\hline CKDcre $($ absence $=0$, presence $=1)$ & $1.66(0.70-3.93)$ & 0.257 & $1.52(0.81-2.88)$ & 0.204 \\
\hline CKDcys $($ absence $=0$, presence $=1$ ) & $4.18(1.81-9.66)$ & $<0.001$ & $2.65(1.39-5.07)$ & 0.005 \\
\hline eGFRcys/eGFRcre $<1.0($ absence $=0$, presence $=1)$ & $3.53(1.47-8.48)$ & 0.003 & $1.72(0.95-3.09)$ & 0.076 \\
\hline
\end{tabular}


Table 5 Multivariate logistic regression analysis of factors associated with sarcopenia in men (A), in women (B)

\begin{tabular}{|c|c|c|c|c|c|c|c|c|}
\hline \multirow[b]{2}{*}{ Variables } & \multicolumn{2}{|l|}{ Model 1} & \multicolumn{2}{|l|}{ Model 2} & \multicolumn{2}{|l|}{ Model 3} & \multicolumn{2}{|l|}{ Model 4} \\
\hline & OR $(95 \% \mathrm{CI})$ & $p$ value & OR $(95 \% \mathrm{CI})$ & $p$ value & OR $(95 \% \mathrm{CI})$ & $p$ value & OR $(95 \% \mathrm{CI})$ & $p$ value \\
\hline \multicolumn{9}{|l|}{ (A) Men } \\
\hline Age (per 1SD) & $1.38(0.88-2.16)$ & 0.154 & $1.31(0.83-2.08)$ & 0.243 & $1.11(0.67-1.84)$ & 0.674 & $1.15(0.70-1.89)$ & 0.590 \\
\hline BMI (per 1SD) & $0.29(0.16-0.53)$ & $<0.001$ & $0.26(0.14-0.50)$ & $<0.001$ & $0.27(0.15-0.51)$ & $<0.001$ & $0.32(0.18-0.58)$ & $<0.001$ \\
\hline $\mathrm{Hb}($ per 1SD) & $1.16(0.73-1.85)$ & 0.517 & $1.21(0.76-1.94)$ & 0.400 & $1.19(0.76-1.86)$ & 0.434 & $1.11(0.71-1.73)$ & 0.652 \\
\hline Alb (per 1SD) & $0.46(0.28-0.78)$ & 0.003 & $0.48(0.29-0.82)$ & 0.005 & $0.50(0.29-0.85)$ & 0.010 & $0.46(0.27-0.79)$ & 0.004 \\
\hline eGFRcre (per 1SD) & & & $0.74(0.44-1.26)$ & 0.263 & & & & \\
\hline eGFRcys (per 1SD) & & & & & $0.60(0.35-1.05)$ & 0.069 & & \\
\hline eGFRcys/eGFRcre (per 1SD) & & & & & & & $0.62(0.34-1.17)$ & 0.135 \\
\hline \multicolumn{9}{|l|}{ (B) Women } \\
\hline Age (per 1SD) & $2.55(1.86-3.51)$ & $<0.001$ & $2.71(1.93-3.81)$ & $<0.001$ & $2.42(1.68-3.47)$ & $<0.001$ & $2.31(1.67-3.20)$ & $<0.001$ \\
\hline BMI (per 1SD) & $0.46(0.32-0.66)$ & $<0.001$ & $0.46(0.32-0.67)$ & $<0.001$ & $0.45(0.31-0.65)$ & $<0.001$ & $0.44(0.30-0.63)$ & $<0.001$ \\
\hline $\mathrm{Hb}$ (per 1SD) & $1.17(0.85-1.61)$ & 0.320 & $1.16(0.84-1.60)$ & 0.363 & $1.18(0.86-1.62)$ & 0.297 & $1.17(0.85-1.62)$ & 0.332 \\
\hline Alb (per 1SD) & $0.98(0.69-1.39)$ & 0.901 & $0.99(0.70-1.40)$ & 0.939 & $0.98(0.69-1.40)$ & 0.938 & $1.04(0.73-1.48)$ & 0.834 \\
\hline eGFRcre (per 1SD) & & & $1.18(0.84-1.67)$ & 0.332 & & & & \\
\hline eGFRcys (per 1SD) & & & & & $0.89(0.62-1.29)$ & 0.540 & & \\
\hline eGFRcys/eGFRcre (per 1SD) & & & & & & & $0.64(0.44-0.93)$ & 0.014 \\
\hline
\end{tabular}

Model 1 included age, BMI, hemoglobin and albumin as covariates. In other models, eGFRcre (model 2), eGFRcys (model 3) and eGFRcys/ eGFRcre ratio (model 4) were added to model 1

Table 6 Multivariate logistic regression analysis of CKDcre, CKDcys and eGFRcys/eGFRcre $<1.0$ adjusted for complications (hypertension, diabetes, dyslipidemia, liver disease, and heart disease), associated with sarcopenia in men (A), in women (B)

\begin{tabular}{llr}
\hline Variables & OR $(95 \% \mathrm{CI})$ & $p$ value \\
\hline (A) Men & & \\
CKDcre & $1.63(0.67-3.93)$ & 0.287 \\
CKDcys & $4.88(2.00-11.95)$ & $<0.001$ \\
eGFRcys/eGFRcre $<1.0$ & $3.70(1.52-9.00)$ & 0.003 \\
(B) Women & & \\
CKDcre & $1.68(0.88-3.22)$ & 0.129 \\
CKDcys & $2.67(1.37-5.19)$ & 0.006 \\
eGFRcys/eGFRcre $<1.0$ & $1.50(0.82-2.74)$ & 0.196 \\
\hline
\end{tabular}

Participants with high muscle mass are likely to have high creatinine levels and low eGFRcre, and participants with sarcopenia are likely to have low creatinine levels and high eGFRcre. In the elderly sarcopenia patients, renal function is overestimated by using eGFRcre. eGFRcys reflects the renal function more accurately than eGFRcre. The relationship between sarcopenia and renal function is established by estimating renal function using eGFRcys.

Second, this study showed the eGFRcys/eGFRcre ratio was significantly positively correlated to SMI, SMM, and muscle strength and physical funtion parameters and was negatively correlated to BFM and percentage of BFM. (Table 3). The eGFRcys/eGFRcre ratio may provide a clinically relevant measurement of muscle mass, based on the assumption that eGFRcre is determined using not only renal function but also muscle mass [5]. To the best of our knowledge, this study is the first study which shows that the eGFRcys/eGFRcre ratio may be a clinically useful parameter for reduced muscle mass in community-dwelling elderly individuals.

The correlation coefficients between eGFRcys/eGFRcre and muscle volume and strength parameters were quite low in this study. In our previous study, the correlation coefficients between $\mathrm{Cr} / \mathrm{CysC}$ and said parameters were observed to be quite low. For example, the correlation coefficients (r) between $\mathrm{Cr} / \mathrm{CysC}$ and SMI were $r=0.34(p<0.0001)$ in men and $r=0.08(p=0.0767)$ in women [2]. Other studies reported the correlation coefficients between $\mathrm{Cr} / \mathrm{CysC}$ and muscle volume, they were also not so high. Barreto et al. reported, $\mathrm{Cr} / \mathrm{CysC}$ correlated with muscle volume evaluated with an abdominal CT scan. The correlation coefficients $(r)$ between $\mathrm{Cr} / \mathrm{CysC}$ and muscle volume was also quite low $(r=0.40)$ [17]. Kashani $\mathrm{K}$ et al. reported $\mathrm{Cr} / \mathrm{CysC}$ correlated with muscle volume in lung transplant candidates, and the correlation coefficients $(r)$ were also the same level [18].

Although there are many reports $\mathrm{Cr} / \mathrm{CysC}$ is associated with muscle volume loss [17-23], we can not estimate the muscle volume or muscle power and diagnosis sarcopenia by only $\mathrm{Cr} / \mathrm{CysC}$. This study showed, not only $\mathrm{Cr} / \mathrm{CysC}$ but also eGFRcys/eGFRcre surely related to muscle volume and muscle power parameters. Because the eGFRcys and eGFRcre measurements are routinely carried out in the clinical 
setting of managing patients with CKD, our results strongly suggest that there is a possibility that eGFRcys/eGFRcre is useful as a screening tool of sarcopenia. Further investigations are needed for more sophisticated screening tools by using these parameters.

Third, this study showed that in participants with sarcopenia, an eGFRcys/eGFRcre ratio $<1.0$ is more frequent than in normal participants (Table 2). In the multivariate logistic regression analysis adjusted for complications, an eGFRcys/ eGFRcre ratio $<1.0$ was clearly shown to be associated with sarcopenia in men (Table 6).

In the ROC analysis, the cut-off value of the eGFRcys/ eGFRcre ratio was 1.00 in men and 1.09 in women. Although the 1.0 cut-off value of the eGFRcys/eGFRcre ratio is easy to evaluate, it may be too low for women. This discrepancy of the cut-off value between men and women may explain the reason why the eGFRcys/eGFRcre ratio $<1.0$ found only in men was clearly shown to be associated with sarcopenia (Fig. 1).

The AUC of eGFRcys/eGFRcre was higher in men (0.693) than in women (0.630). Similarly, in a previous study, the AUC of $\mathrm{Cr} / \mathrm{CysC}$ for identifying sarcopenia was higher in men than in women [23]. Generally, total muscle volume is higher in men than in women. The influence of the change in muscle volume is less in CysC than in Cre. Therefore, the change in eGFRcys/eGFRcre by the decrease in skeletal muscle mass is expected to be bigger in men than in women. The difference in body composition between sexes and the discrepancy in the influence on eGFRcys/eGFRcre by the change in muscle volume between men and women may explain said difference.

There are no participants with CKD stage 5 in both sexes. This result indicates that even in the participants without end-stage renal failure, only "eGFRcre is more than eGFRcys" may be related to sarcopenia. Recently it has been reported that difference between eGFRcre and eGFRcys (dGFR) correlates with muscle strength positively in patients with liver damage [24]. The eGFRcre may become detached from eGFRcys in patients with sarcopenia.

The release of creatine from the muscle is the major determinant of serum $\mathrm{Cr}$ levels, due to its conversion to $\mathrm{Cr}$ in the circulation. CysC is a cysteine protease inhibitor that is constantly produced by all nucleated cells. Thus, it is unaffected by muscles mass [5] and the eGFRcys value has a lower level of bias. As mentioned in a previous study, CysC may be influenced by mild chronic inflammation and oxidative stress $[25,26]$. Thus, eGFRcys may be more sensitive to mild inflammatory and oxidative changes in sarcopenia than eGFRcre. Recently, a significant relationship was observed between the risk for CVD and sarcopenia, because of the increased circulating markers of oxidative stress in sarcopenia [27]. These mechanisms may support our present study. In Japan, CysC levels are widely monitored in daily clinical settings. Moreover, in numerous institutions, both eGFRcre and eGFRcys are calculated automatically by the center clinical inspection section. An eGFRcys/eGFRcre ratio $<1.0$ can be evaluated easily in many institutions.

This study has some limitations that must be considered. First, this is a cross-sectional study. Therefore, any causeand-effect relationships cannot be evaluated. A prospective study must be conducted to assess any causal associations between CKD and sarcopenia. Second, most of the participants in this study voluntarily participated in our study. Thus, the study participants may be healthier, and the study population might have lower rates of sarcopenia than those in the general population. This could account for any inconsistency between our results and previous studies. Third, we did not measure urinary protein. Thus, the association between CKD and sarcopenia that was modified by the presence of subclinical kidney disease was not examined. Finally, a small number of participants with sarcopenia were included in the study, which obviously limits the reliability and applicability of the proposed test.

In conclusion, CKDcys but not CKDcre is associated with sarcopenia. A low eGFRcys/eGFRcre ratio may be a practical screening marker for sarcopenia based on the AWGS 2019 criteria in rural community-dwelling older adults. Further studies are needed to evaluate the diagnostic value of the eGFRcys/eGFRcre ratio for estimating sarcopenia. The prognostic value of the eGFRcys/eGFRcre ratio for predicting clinical outcomes in older adults also warrants further study.

Acknowledgements We would like to thank all the medical staff of Sasayama Medical Center Hyogo College of Medicine who supported the FESTA study.

Funding This study was supported in part by JSPS KAKENHI (grant number: 16KT0012 (2016-2018) and 19K16995 (2019-2022)), the Medical Research Fund by Hyogo Medical Association (2016), a grant for the support of collaborative investigation between Hyogo College of Medicine and Hyogo University of Health Sciences (2017-2019) (Dr. Shinmura and Dr. Kusunoki), and the grant for Good Practice to Establish Centers for Fostering Medical Researchers of the Future by Ministry of Education, Culture, Sports, Science and Technology (2015-2017) (Hyogo College of Medicine).

\section{Compliance with ethical standards}

Conflict of interest The authors have declared that no conflict of interest exists.

Ethical approval All procedures performed in studies involving human participants were in accordance with the ethical standards of the institutional and/or national research committee at which the studies were conducted (IRB approval number Rinhi 0342 at Hyogo College of Medicine) and with the 1964 Helsinki declaration and its later amendments or comparable ethical standards. 
Informed consent Informed consent was obtained from all individuals participants included in the study.

Open Access This article is licensed under a Creative Commons Attribution 4.0 International License, which permits use, sharing, adaptation, distribution and reproduction in any medium or format, as long as you give appropriate credit to the original author(s) and the source, provide a link to the Creative Commons licence, and indicate if changes were made. The images or other third party material in this article are included in the article's Creative Commons licence, unless indicated otherwise in a credit line to the material. If material is not included in the article's Creative Commons licence and your intended use is not permitted by statutory regulation or exceeds the permitted use, you will need to obtain permission directly from the copyright holder. To view a copy of this licence, visit http://creativecommons.org/licenses/by/4.0/.

\section{References}

1. Hiraki K, Yasuda T, Hotta C, Izawa KP, Morio Y, Watanabe S, et al. Decreased physical function in pre-dialysis patients with chronic kidney disease. Clin Exp Nephrol. 2013;17(2):225-31.

2. Kusunoki H, Tsuji S, Wada Y, Fukai M, Itoh M, Sano K, et al. Relationship between sarcopenia and the serum creatinine/cystatin $\mathrm{C}$ ratio in Japanese rural community-dwelling older adults. $\mathrm{J}$ Cachex Sarcopenia Muscle-Clin Rep. 2018;3:e00057.

3. Osaka T, Hamaguchi M, Hashimoto Y, Ushigome E, Tanaka M, Yamazaki M, et al. Decreased the creatinine to cystatin $\mathrm{C}$ ratio is a surrogate marker of sarcopenia in patients with type 2 diabetes. Diabetes Res Clin Pract. 2018;139:52-8.

4. Dalrymple LS, Katz R, Rifkin DE, Siscovick D, Newman AB, Fried LF, et al. Kidney function and prevalent and incident frailty. Clin J Am Soc Nephrol. 2013;8(12):2091-9.

5. Baxmann AC, Ahmed MS, Marques NC, Menon VB, Pereira AB, Kirsztajn GM, et al. Influence of muscle mass and physical activity on serum and urinary creatinine and serum Cystatin C. Clin J Am Soc Nephrol. 2008;3(2):348-54.

6. Kurajoh M, Inaba M, Nagata Y, Yamada S, Imanishi Y, Emoto M. Association of cystatin C- and creatinine-based eGFR with osteoporotic fracture in Japanese postmenopausal women with osteoporosis: sarcopenia as risk for fracture. J Bone Miner Metab. 2019;37(2):282-91.

7. Miyakoshi N, Hongo M, Mizutani Y, Shimada Y. Prevalence of sarcopenia in Japanese women with osteopenia and osteoporosis. J Bone Miner Metab. 2013;31(5):556-61.

8. Go SW, Cha YH, Lee JA, Park HS. Association between sarcopenia, bone density, and health-related quality of life in Korean men. Korean J Fam Med. 2013;34(4):281-8.

9. Yoshimura N, Muraki S, Oka H, Iidaka T, Kodama R, Kawaguchi $\mathrm{H}$, et al. Is osteoporosis a predictor for future sarcopenia or vice versa? Four-year observations between the second and third ROAD study surveys. Osteoporos Int. 2017;28(1):189-99.

10. Foundation NK. K/DOQI clinical practice guidelines for chronic kidney disease: evaluation, classification, and stratification. Am J Kidney Dis. 2002;39(2 Suppl 1):S1-266.

11. Matsuo S, Imai E, Horio M, Yasuda Y, Tomita K, Nitta K, et al. Revised equations for estimated GFR from serum creatinine in Japan. Am J Kidney Dis. 2009;53(6):982-92.

12. Horio M, Imai E, Yasuda $Y$, Watanabe $T$, Matsuo $S$, GFR CDTJEFE. GFR estimation using standardized serum cystatin C in Japan. Am J Kidney Dis. 2013;61(2):197-203.
13. Chen LK, Woo J, Assantachai P, Auyeung TW, Chou MY, Iijima K, et al. Asian Working Group for Sarcopenia: 2019 consensus update on sarcopenia diagnosis and treatment. J Am Med Dir Assoc. 2020;21(3):300-7.

14. Shastri S, Tighiouart H, Katz R, Rifkin DE, Fried LF, Shlipak MG, et al. Chronic kidney disease in octogenarians. Clin J Am Soc Nephrol. 2011;6(6):1410-7.

15. Peralta CA, Katz R, Sarnak MJ, Ix J, Fried LF, De Boer I, et al. Cystatin $\mathrm{C}$ identifies chronic kidney disease patients at higher risk for complications. J Am Soc Nephrol. 2011;22(1):147-55.

16. Liu CK, Lyass A, Massaro JM, D’Agostino RB, Fox CS, Murabito JM. Chronic kidney disease defined by cystatin $\mathrm{C}$ predicts mobility disability and changes in gait speed: the Framingham Offspring Study. J Gerontol A Biol Sci Med Sci. 2014;69(3):301-7.

17. Barreto EF, Poyant JO, Coville HH, Dierkhising RA, Kennedy $\mathrm{CC}$, Gajic $\mathrm{O}$, et al. Validation of the sarcopenia index to assess muscle mass in the critically ill: A novel application of kidney function markers. Clin Nutr. 2019;38(3):1362-7.

18. Kashani K, Sarvottam K, Pereira NL, Barreto EF, Kennedy CC. The sarcopenia index: a novel measure of muscle mass in lung transplant candidates. Clin Transpl. 2018;32(3):e13182.

19. Kashani KB, Frazee EN, Kukralova L, Sarvottam K, Herasevich $\mathrm{V}$, Young PM, et al. Evaluating muscle mass by using markers of kidney function: development of the sarcopenia index. Crit Care Med. 2017;45(1):e23-9.

20. Yanishi M, Kinoshita H, Tsukaguchi H, Kimura Y, Koito Y, Sugi $\mathrm{M}$, et al. The creatinine/cystatin $\mathrm{C}$ ratio provides effective evaluation of muscle mass in kidney transplant recipients. Int Urol Nephrol. 2019;51(1):79-83.

21. Tabara Y, Kohara K, Okada Y, Ohyagi Y, Igase M. Creatinineto-cystatin $\mathrm{C}$ ratio as a marker of skeletal muscle mass in older adults: J-SHIPP study. Clin Nutr. 2020;39(6):1857-62.

22. Lin YL, Chen SY, Lai YH, Wang $\mathrm{CH}$, Kuo $\mathrm{CH}$, Liou HH, et al. Serum creatinine to cystatin $\mathrm{C}$ ratio predicts skeletal muscle mass and strength in patients with non-dialysis chronic kidney disease. Clin Nutr. 2020;39(8):2435-41.

23. He Q, Jiang J, Xie L, Zhang L, Yang M. A sarcopenia index based on serum creatinine and cystatin $\mathrm{C}$ cannot accurately detect either low muscle mass or sarcopenia in urban community-dwelling older people. Sci Rep. 2018;8(1):11534.

24. Ichikawa T, Miyaaki H, Miuma S, Motoyoshi Y, Yamashima M, Yamamichi $\mathrm{S}$, et al. Indices calculated by serum creatinine and cystatin $\mathrm{C}$ as predictors of liver damage, muscle strength and sarcopenia in liver disease. Biomed Rep. 2020;12(3):89-98.

25. Xu Y, Ding Y, Li X, Wu X. Cystatin C is a disease-associated protein subject to multiple regulation. Immunol Cell Biol. 2015;93(5):442-51.

26. Muslimovic A, Tulumovic D, Hasanspahic S, Hamzic-Mehmedbasic A, Temimovi R. Serum cystatin C - marker of inflammation and cardiovascular morbidity in chronic kidney disease stages 1-4. Mater Sociomed. 2015;27(2):75-8.

27. Bellanti F, Romano AD, Lo Buglio A, Castriotta V, Guglielmi $\mathrm{G}$, Greco A, et al. Oxidative stress is increased in sarcopenia and associated with cardiovascular disease risk in sarcopenic obesity. Maturitas. 2018;109:6-12.

Publisher's Note Springer Nature remains neutral with regard to jurisdictional claims in published maps and institutional affiliations. 